\title{
How and Why Finnish Universities of Applied Sciences Defined a New Approach to Build a Next Generation Student and Study Management System
}

\author{
Tuulikki Paturi \\ HAAGA-HELIA University of Applied Sciences, Finland
}

\begin{abstract}
This paper reports a project where Finnish Universities of Applied Sciences (UAS) defined a new approach to build a next generation Student and Study Management System (SSMS). The project identified, defined and described the key processes in a future SSMS. The definition based on business processes described by expert users. The project developed tools and rules to draw and read the process charts.
\end{abstract}

Keywords: Student and Study Management System (SSMS), business process definition, process description.

\section{BACKGROUND}

The Finnish Universities of Applied Sciences, UAS, (former polytechnics) have operated from the mid-1990's. Each UAS introduced an own SSMS at that time. The systems have been developed continuously but they are based on client-server architecture and their operational logic has its origin in the outdated techniques of that time. They do not satisfy in all respects the information needs of universities any more and few or none educational planning processes are supported. The present systems register the transactions well but do not support resource planning nor are the present systems in accordance with the linings of the information management strategy of the Finnish Ministry of Education.

The Ministry of Education has published a strategy for information management in years $2006-2015$ (2006). The strategy has a vision:

As a result of cooperation in information management the administrative sector has well defined concepts, business processes and interfaces which have helped to build innovative integrated and dedicated services for the sector. This leads to efficient operation and

Please use the following format when citing this chapter:

Paturi, T., 2009, in IFIP International Federation for Information Processing, Volume 292; Evolution of Information Technology in Educational Management; Eds. Tatnall, A., Visscher, A., Finegan, A., O’Mahony, C., (Boston: Springer), pp. 117-128. 
services in education, research and culture are available in a uniform and easy way.

The strategy has two significant linings that met the needs of UAS:

1. Strengthening of the common basic information about teaching, research and culture and electronic services.

The objective is to improve the information support of studying, teaching, research and culture, to increase the integrated use of the information and to reduce the number of separate systems. For this, the core concepts and basic processes concerning the students, study records and teaching are unified and they serve as a basis for the common information processing systems which include the whole process from application up to the completion of studies. All the new services are carried out with electronic services.

2. The common architecture of information management and services In the services of the administrative sector, the architectural linings of the public administration, defined standards and open interfaces are carried out. The basis for the system development is service oriented architecture (SOA) which makes the effective use of core functions and databases possible also in unconnected applications.

These reasons led to the fact that ARENE (the Rectors' Conference of Finnish Universities of Applied Sciences) established the project ProAMK (Pro UAS) to specify the functionality of the student and study management system for Finnish Universities of Applied Sciences. The project started in the autumn of 2005 and ended December 2007. The project was financed by the Ministry of Education and by the UAS equally, and the costs were 60000 Euro.

\section{ORGANISATION OF THE PROJECT PROAMK}

Based on preliminary work ARENE named a steering group for the project and a project group of eight experts as well. Each expert represented a certain field in educational management within UAS. They were nominated by ARENE after a survey of willingness to participate. The project group was supported by five expert groups in different fields in educational management. The project had a full-time project manager. A total of 29 UAS were represented in the expert groups by 250 experts in educational and administrative management. The project used Moodle learning environment as working space and all documents were published there for those interested to follow the proceedings (Project ProAMK 2007). 


\section{THE PROJECT OBJECTIVES}

The future information system will be used as the most central information system of the educational management. All the information which is necessary for the planning, operating, reporting and evaluating a university in action is registered in it. The project has three main objectives

1. To identify and define the processes which are common to all the actors and are described on the basis of the present state. On the basis of these the core level of the system is defined.

2. To identify and describe the processes which are common to every UAS and which are the key processes to be supported by the new SSMS.

3. To define the models, types of functionalities and interfaces for UAS to enable other information systems to operate as a part of the new SSMS. These can be for instance payroll systems or do-it-yourself systems for specialized needs.

(Project ProAMK 2007)

What kind of a future does the new SSMS serve? (Stenlund 2007)

- From a registration system to a resource planning system.

- Versatile (funding bodies, administrator(s), customers, students, management).

- Time conscious; changes in the structures are possible and expected.

- It always supports the objectivity; from the individual level to ministry level.

- Supports evaluation and prognoses; from objective definition to the outcomes.

- Both the social mission and the financing model place the students' success to realize their own plans at the core of the matter.

\section{OPERATIONAL ENVIRONMENT}

The project group first identified all the actors involved in the UAS and what kind of functionality they represent in the year around life of the UAS. The result is depicted in Figure 1 (Ruuskanen, Hyttinen 2007). 


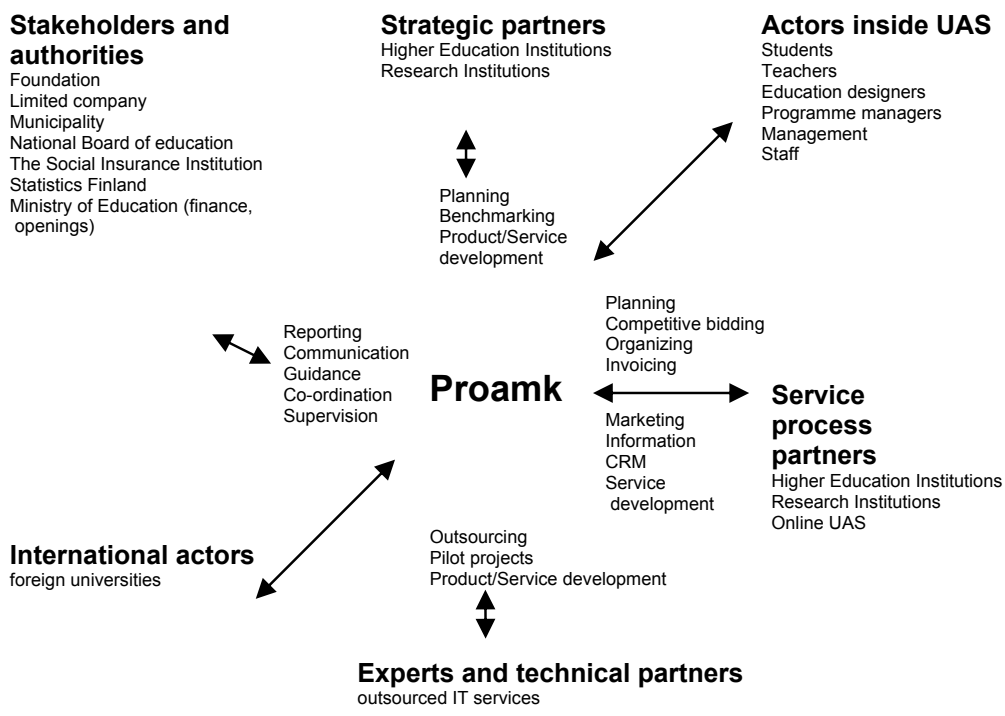

Figure 1: The UAS operational environment

\section{WHAT PROCESSES SHOULD AN SSMS INCLUDE?}

At first the project group gathered together in order to define the most essential core processes and supporting processes in SSMS. After many sessions, the result is depicted in the following Figure 2 (Ruuskanen, Hyttinen 2007).

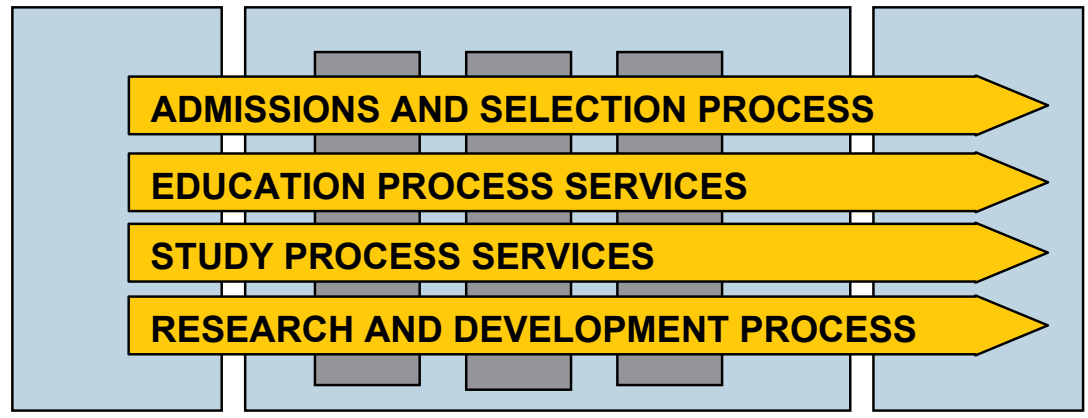

Figure 2: Core processes and supporting processes in SSMS

The core processes of an SSMS are service processes which support both the core processes and the supporting processes of a University of Applied Sciences. The core processes are the following: Admissions and selection process, Education process, Study process and Research and development 
process. The supporting processes are User services, Student services and Report services.

This approach is at so high a level that every UAS could accept it. While defining the sub processes we found the difficulty of speaking the same language. All the core concepts did not have the same content and the processes were managed differently in every UAS. The actors in the processes differed in their organisational status and this was a common fact to confuse the mutual understanding about the process content. Soon we found out that we need tools to success in the SSMS definition. The tools were the selection of a standard notation for process descriptions and the definition of the concepts.

\section{HOW TO ACHIEVE JOINT UNDERSTANDING IN PROCESS DESCRIPTIONS?}

One of the most significant tasks of the definition phase was to describe the processes of the basic operation of UAS so that they give a good base to continue to the system specification. Each member of the project group was responsible for one big process and together with the expert groups they divided the processes into sub processes.

The project group studied earlier implementations of process definitions by users and learned the need for easy tools to draw the processes and the need for well-specified notations. The project group chose MS Visio to be the drawing tool mainly because every UAS had MS Visio due to the contract between Microsoft and Finnish higher education institutions. The processes were described using BPMN (Business Plan Model Notation) Swim lane charts and the project group guided the drawing by defining a template, choosing a stencil and making rules for process elements. The stencil included all the necessary and the only allowed symbols. The rules were simple:

- The actor at the end of the swim lane is universal and reveals only the role of the actor, not the organisational position. Each UAS can conjure up their professionals as actors.

- The process proceeds with the model input - process - output. Every activity is followed by an output which serves as an input to the next activity. This was the key success factor in managing the thinking of several people in several organisations with different backgrounds.

After describing the processes, each project group member demonstrated the charts to his/her expert group. The discussion in expert groups was stormy and revealed the need for concept definitions. The project group decided not to define every concept within a UAS (= never ending task). Instead the concept definition was strictly restricted to those concepts needed within the SSMS. These concepts were found in the process 
descriptions as data objects and were defined from the point of view of the information system. Every process description has a user story to help the decoding of the chart. The next Figure (3) depicts one example of 45 produced process descriptions.

\begin{tabular}{|l|l|}
\hline $\begin{array}{l}\text { E. Study process services } \\
\text { E8. Graduation }\end{array}$ \\
\hline -
\end{tabular}

Figure 3: Example of a process description (Project ProAmk 2007)

The project manager organized a wide road show (7 meetings) to introduce the processes to about 250 experts in UAS. Afterwards the descriptions were circulated for official expert comments to the UAS at the end of 2006. The first round generated so many comments that the steering group decided to have a second circulation for comments at the beginning of 
2007. The process descriptions were updated according to all relevant comments.

\section{DEFINING THE CONCEPTS}

The terminology work was begun after the production of the first versions of the process descriptions. The project group produced a lexicon of about 180 terms, their concepts and with references to other lexicons. All terms used in the process descriptions were restricted to be in the scope of the SSMS to be defined. The project group did not try to define all the terms within the sector of educational administration and management because the specified definitions are related to the context they are used in. This fact was very important to realize and it clarified the discussions and definitions.

\section{FOREIGN REFERENCES}

The project group decided to take as foreign references the extensive cooperation projects that have been carried out in other Nordic countries and Germany. These systems were LADOK in Sweden, Felles Studentsystem in Norway, STADS in Denmark, SAP and HIS in German. The German system HISinOne is currently under heavy development and it proved to be the only one to meet the strategic linings of Finnish Ministry of Education.

\section{THE RESULTS}

The project succeeded well in identifying, defining and describing the key processes in a Student and Study Management System for Universities of Applied Sciences. These are a good starting point to develop a new SSMS of the future university.

The key processes and their sub processes to be included in the future SSMS are the following:

1. Education process services

- Planning of the coming education

- Planning of the coming courses

- Planning of teachers workloads and timetables

2. User Services

- Students

- Staff

- Student union

- Alumni

3. Student admission and selection services

- Making public the programmes and their entrant places 
- Managing the applications

- Student selection

4. Student services

- Managing the right to study

- Managing changes in the right to study

- Student self-service

- Student financial aid

5. Study process services

- Career planning

- Participating in courses

- Finding a way to enrol for courses in partner HEI's

- Enrolling for exams

- International mobility

- Work placement

- Thesis

- Graduation

6. Reporting services

- Support for quality management (course feedback, programme feedback)

- Reports of all activities inside SSMS

- Data transfers to other information systems

7. Research and development services

- R\&D activities with results

- R\&D reporting

- Project management

(Project ProAMK 2007)

These processes are common to all Universities of Applied Sciences. Other processes like facility management, human resource management and financial management were left out because they depend on the status of the UAS ownership. Municipal UAS use the municipality's information systems and private UAS have their own systems for resources, finance and payrolls.

The vision of the information technology in educational management within higher education is presented in Figure 4. It is based on the ideas of electronic services and service oriented architecture.

The operational system has core functions that may have been implemented with IT systems from different software producers. The only requirement is that these systems can change data by integrating them together via open interfaces. A layer of service process applications (in one portal) provide process oriented web services to the user.

The interface to the system is so well designed to support the business process that no special training is needed for the users. The users have role dependent views to the system which define the rights to see and update the stored data. User self service is available 24/7.

A data warehouse is attached to the operational system to gather all the relevant information for managing the university. The data warehouse 
includes business intelligence tools (for reporting and analysing), tools to extract, transform, and load data into the repository, and tools to manage and retrieve metadata. The whole repository is used to by the university management and some statistical parts of it can even be used by authorities.

The performance feedback system includes the tools for quality assurance to provide confidence of the effectiveness of the universities functions.

\section{UNIVERSITYITEM}

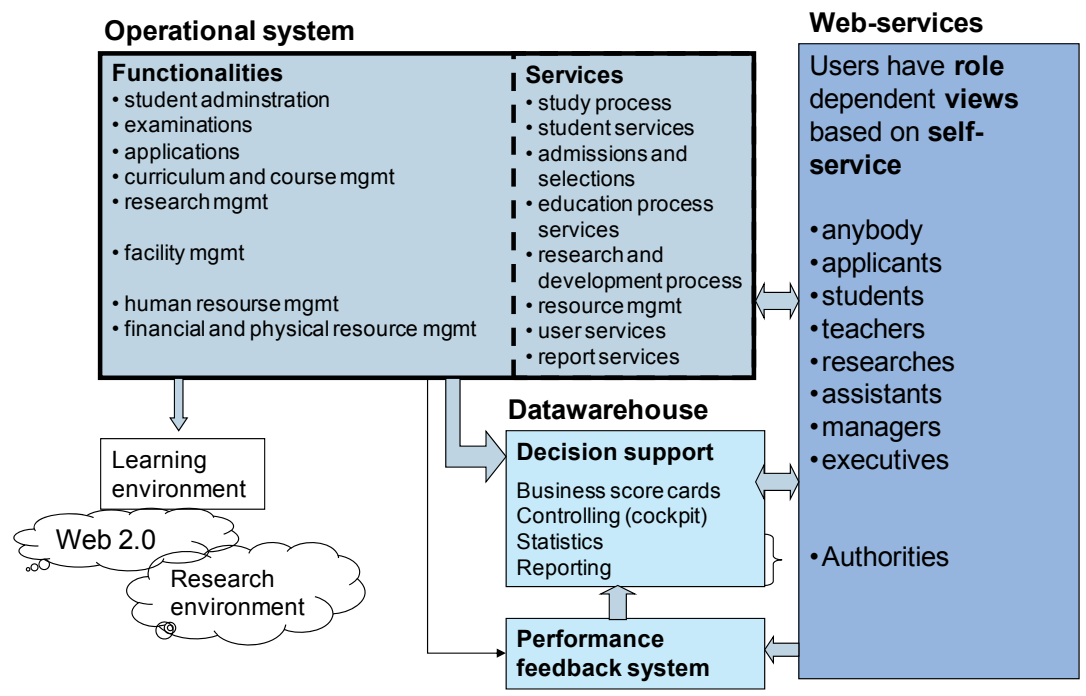

Figure 4: University Information Technology in Educational Management

The project did not succeed in defining the models, types of action and interfaces connecting other information systems to operate as a part of the new SSMS. This definition required a vision of the technical realization of the system and the project group did not reach consensus in this matter. The interest groups and comparison of alternatives were too many and the amount of work needed to reach a consensus did not meet the deadline. The open questions were among others the way to organize the building of the system, should the database be centralized or decentralized, should the system be built from a scratch or is there a ready made system that fulfills the objectives, what is the role of UAS and its DIY-systems connected to the current SSMS an so on.

During the project period the traditional universities realized that they have same kind of problems and they showed some interest to start a similar project. In a country of 5 million people it is neither wise nor cost-effective to build two separate SSMS in higher education. The steering group decided not to give more time to the ProAMK project. Instead they outlined a new approach to the problem area and a new project is starting to continue the work together with the traditional universities, Ministry of Education and 
CSC. (CSC is the Finnish IT center for science, a non-profit company administered by the Ministry of Education providing IT support and resources for academia, research institutes and companies).

\section{THE FUTURE}

The Ministry of Education started in March 2008 a project to carry out three master projects:

1. A model of Enterprise Architecture for HEI

2. Definition of concepts, common concept model and a common data warehouse

3. A generic SSMS for all HEIs.

\subsection{A model of Enterprise Architecture for HEI Information Technology}

The Ministry of Education proposes that a description of functions with supporting information systems to be developed for higher education institutions according to the model of Enterprise Architecture developed for Finland's public administration. Enterprise architecture is a tool for developing the organisation and its information systems. It is used for collaboration between top management and chief information officers (Pulkkinen, Valtonen, Heikkilä, Liimatainen 2007).

The model of enterprise IT architecture defines responsibilities and common functional models at the following levels:

- Functional architecture

The services and processes from the point of view of the life cycle of the users and objects and from the point of view of administrative structures and the social role.

- Information architecture

The concepts which are related to functionalities, the production of services and processes, the data elements corresponding to the concepts, the structures of the concepts and data relations between them and the relations to the processes and services.

- IT-system architecture

The services produced by the information systems and the information processing support for functionality and the relations between the information systems and data.

- Technology architecture

The technology related solutions for IT-systems and ITinfrastructure and the structure of IT-systems. 


\subsection{Definition of concepts, common concept model and a data warehouse}

This project will produce a data warehouse to be shared within all HEIs and the project will take into account the outcomes of the ProAMK-project and other releated previous projects and data warehouse solutions as well. The Ministry of Education reserves the right to define the concepts needed for governing HEIs.

\subsection{A generic SSMS for all HEIs}

A cooperative project between the traditional universities and universities of applied sciences will be started with following actions:

- Define and implement a generic SSMS (uniform knowledge and uniform basic processes).

- The University of Kuopio started a project to report the essential functional similarities and differences in current SSMS.

- The applicability for Finnish circumstances and the quality of the HISinOne system currently under development by German nonprofit organization Hochschule-Informations-System $\mathrm{GmbH}$ will be evaluated.

HISinOne is web-based integrated software system that covers all business processes of universities of any size and form of organization and supports the requirements of the Bologna process. It uses service-oriented architecture with interconnected components. Interoperability with existing environments is possible with webservices, integration of databases and import/export functions. HIS is an open source developing community.

- The definition of study administrative processes will continue based on the ProAMK project.

- The existing admission and student selection systems within HEI will be recognized.

CSC, the Finnish IT center for science, will coordinate these projects and the next checkpoint will be on November 2008 with further decisions.

During the ProAMK period the surrounding world changed so that the project can now be considered as the first step to realize a new SSMS for Finnish HEI. All the agreements of objectives and results for the year 2009 between the Ministry of Education and universities (traditional and UAS) include the commitment to participate in these three master projects explained above. 


\section{REFERENCES}

HISinOne Die neue Software-Generation 2008 http://www.his.de/pdf/01/ HISinOneInformationsbroschuere.pdf. (with English summary) HIS Hochschule-Informations-System GmbH: http://www.his.de/english/ organization, Date accessed 30.5.2008

Ministry of Education (2006): The strategy for information management in years 2006 - 2015 in the administrative sector of Ministry of Education, Helsinki Finland, ISBN 952-485-260-8

Project ProAMK (2007) documentation on web-page https://www.ProAMK. fi/ (in Finnish), Date accessed 30.5.2008

Pulkkinen, Valtonen, Heikkilä, Liimatainen 2007: Enterprise architecture models, Finnish Enterprise Architecture Research, Project Research and studies 3/2007, Ministry of Finance, June 2007 ISBN 978-951-804713-4

Ruuskanen, Hyttinen (2007) Proamk - loppuraportin tiivistelmä 17.1.2007 WM-data Oy, https://www.proamk.fi/raportit/Proamk-v1.0.pdf, part of Project ProAMK documentation, Date accessed 30.5.2008

Stenlund Thomas (2007) Tietoperusteinen johtaminen ammattikorkeakoulukontekstissa, Ineo Corporate Performance Management, part of Project ProAMK documentation, Date accessed 30.5.2008 